\title{
SocArXiv
}

Preprint : November 19, 2021

\section{Online Social Integration and Depressive Symptoms in Adolescents}

\author{
Elizaveta Sivak \\ HSE University \\ esivak@hse.ru
}

\author{
Julia Dementeva \\ HSE University \\ yudementeva@hse.ru
}

\author{
Ivan Smirnov \\ HSE University \\ ibsmirnov@hse.ru
}

\begin{abstract}
Social integration is known to be beneficial for mental health. However, it is not clear whether this applies to online as well as offline relationships. In this paper, we explore the association between online friendship and symptoms of depression among adolescents. We combine data from the popular social networking site with survey data on high school students $(N=144)$ and find that integration into the online network is a protective factor against depression. We also find that not all online connections are equally important: friendship ties with students from the same schools are stronger associated with depression than outside ties. In addition to friendship ties, we explore the effect of online interaction ("likes"). Overall, our results suggest that online relationships are associated with depression as well as offline friendship. However, the effect of more distant online connections is limited, while immediate social environment and peer relationships at school are more important.
\end{abstract}

Keywords: adolescents, depression, digital traces, online social integration, psychological wellbeing, social media, social networks.

\section{Introduction}

Social integration is vital to an individual's well-being. People who have stronger social networks are healthier (Ford et al. 2000; House et al. 1988; Umberson et al. 2010) and live longer (Holt-Lunstad et al. 2010). They are also less likely to be depressed (Berkman 1995; Seeman 1996; Thoits 2011; Rosenquist et al. 2011). Association between social ties and psychological well-being is well established and dates back to Durkheim's work on suicides (Durkheim 1897).

Social environment is especially important in adolescence, as during this period of life adolescents start to spend more time in peer groups and the developmental significance of peers 
increases (Brown et al. 2004; Rubin et al. 2007). Based on various measures of social integration, previous research has shown that integration promotes adolescents' mental health. Protective factors associated with lower risks of depressive mood or depression include feeling connected to parents, peers, or school (Costello et al. 2008), a larger number of friends (Ueno 2005), and high social network centrality (Okamoto et al. 2011). Adolescents who are less socially integrated have more internalizing problems, which are common among depressed children (Liu et al. 2011). During the COVID-19 crisis, isolation in social networks and lack of interaction and emotional support were associated with negative mental health trajectories (Elmer et al. 2020).

As people increasingly use social networking sites (SNSs) for communication and maintaining friendship, researchers focus on online social integration - size and properties of online friendship networks and intensity of online contacts. It is yet not clear whether online interactions are beneficial for well-being as well as offline social ties. Previous research has shown that online social integration is linked to reduced mortality risk from the causes that are strongly related to social factors such as drug overdose and suicide but not from other causes such as cancer (Hobbs et al. 2016). Online integration may enhance access to various forms of support and produce positive psychological states like a sense of belonging and self-worth, which in turn protect against distress (Berkman and Kawachi 2001). Even though a friendship on SNS does not necessarily involve a close connection (Manago et al. 2012), a large number of online friends could remind users of their social ties and boost their self-esteem (Gonzales and Hancock 2011). Users who have more online friends should be more likely to receive likes or comments, which are considered a symbolic token of social approval, inclusion, and support (Martinez-Pecino and Garcia-Gavilán 2019; Mascheroni et al. 2015; Zell and Moeller 2018), and elicit feelings of validation, conferring positive status and regard, and thus leads to positive emotions (Davey et al. 2010; Gunther Moor et al. 2010). Therefore, embeddedness in a large online social network may lower the risk of developing depressive symptoms.

There is evidence that social connectedness on SNSs is related to lower levels of depression and anxiety (Seabrook et al. 2016) and higher subjective well-being (Kim and Lee 2011). Larger networks and larger estimated audiences predicted higher levels of life satisfaction and perceived social support on Facebook (Manago et al. 2012), which in turn is associated with reduced stress and greater well-being (Nabi et al. 2013). Receiving more likes and comments was linked to greater self-esteem and happiness (Zell and Moeller 2018).

On the other hand, on SNSs, the probability of unflattering social comparisons increases, because comparison information, e.g. number of friends or "likes" which may serve as status and popularity cues (Blease 2015), is more salient than offline (Appel et al. 2016; Haferkamp et al. 2012; Vogel et al. 2014) and the presented information is positively skewed (Jordan et al. 2011; Chou and Edge 2012; Qui et al. 2012; Lee-Won et al. 2014).

In turn, negative social comparison on SNS is related to higher levels of emotional distress and depression (Feinstein et al. 2013; Steers et al. 2014; Appel et al. 2016; Lup et al. 2015). This supports the hypothesis, which is based on the relative social evaluation theories of depression, that the SNSs' users may be the more susceptible to causal triggers for depression if they have a large number of online friends (Blease 2015). Larger online networks might mean more opportunities for negative social comparisons.

In this paper, we explore how online social integration is associated with depression symptoms of high school students. We measure social integration using the properties of the ego 
network (its overall size and the size of the densest part of the network) and the intensity of online communication. The study was conducted among students of one high school in Moscow, Russia. We used self-reported depression symptoms, information about friendship obtained using name generator, and data from the popular social networking site VK on online friendship and interactions. We studied the associations between symptoms of depression and 1) the number of online friends and structural properties of a person's network, 2) online interactions - the number of received and sent "likes". We also measure the changes in the strength of the association between "likes" (intensity of communication) and depression.

\section{Methods}

\section{Participants and Procedures}

The study was conducted among students of one high school in Moscow, Russia. The study was approved by the Institutional Review Board. Participation was voluntary. Students from 10th and 11th grades (16-17 years old) were informed about the study and the opportunity to participate. The researchers held group meetings with all students who self-selected to participate, in which they explained the aims of the study and what data would be collected. All participants who decided to participate, as well as one of their parents, signed an informed consent form. Students were informed that their participation was voluntary and that they could stop participation at any time and request to delete the collected data (there were no such requests to date).

The study took place over a 4-month period (November 2017-February 2018). Participants were asked to fill out an online survey at the beginning and at the end of this period. Students could participate in the survey at their own time. Both waves included items that measure depression. In the baseline survey, participants were also asked about friendship. The baseline survey was filled out by 144 students ( $81 \%$ girls), the endline by 78 students. Public data were gathered from profiles on VK with the participants' consent.

\section{Measures}

\section{Depression}

We used the Patient Health Questionnaire scale (PHQ-9), which is used to assess the severity of depressive symptoms (Kroenke et al. 2010) ${ }^{1}$. The scale has been shown to be a valid tool in detecting depression among adolescents across various cultures (Burdzovic Andreas and Brunborg 2017; Tsai et al. 2014; Richardson et al. 2010; Fatiregun and Kumapayi 2014; Ganguly et al. 2013).

We measured depressive symptoms twice. The test-retest reliability coefficient is $0.73(P<$ $\left.10^{-11}\right)$. In the analysis, we used results of the first measurement, as it has fewer missing values. According to the PHQ-9 scale, $10 \%$ of the sample had no symptoms of depression (scored 0-4), 41\% exhibited mild symptoms (5-9), 26\% moderate (10-14), 11.5\% moderately severe (15-19), and $11.5 \%$ severe (20-27). These rates seem unusually high: previous studies

\footnotetext{
${ }^{1}$ https://www.phqscreeners.com/
} 
that used PHQ-9 to measure depression among middle and high school students reported the prevalence of moderately severe/severe depression to be 5-9\% (Burdzovic Andreas and Brunborg 2017; Tsai et al. 2014; Tsehay et al. 2020; Leung et al. 2020). In Russia, there is not enough data on the prevalence of depressive symptoms in adolescents to compare our results to. We attribute this high rate to the fact that lower rates are reported for middle and high school students (11-17 years old) while we focus only on high school students. They are 16-17 years old which is the age when the onset for depressive illnesses peaks (Young et al. 2010). They are also preparing for their final exams and are under high academic pressure. Moreover, the school where the study was conducted is highly selective and partners with a university: once a week students attend classes of their choice at the university. Given all this, the more appropriate reference group might be university students. For that group, the reported depression prevalence is $10-85 \%$ with a weighted mean prevalence of $30.6 \%$ (Ibrahim et al. 2013).

Because of the high rate of students with moderately severe and severe depressive symptoms according to conventional thresholds, we use PHQ-9 severity score as a continuous variable. This severity score is known to be strongly associated with other indicators of depression and mental health, and also with the different aspects of health-related quality of life and can be used in addition to the categorical diagnostic groups (Kroenke et al. 2001).

\section{Online Social Integration}

We used several measures of online integration. For each participant, we measured the degree to which an individual is connected to other individuals in a network, i.e. the number of "friends" they had on VK a) overall, b) among those who studied at the same school. We also measure the intensity of communication as the number of "likes" participants received per post a) overall, b) from students from the same school. Unlike an almost static friendship network (once added, friends are rarely deleted), likes have a temporal dimension, i.e. it is possible to infer when the like was received. This allows us to look at the relationship between the number of likes and symptoms of depression at the time of the measurement of depression and before that (six months before the data collection).

Finally, to understand the potential role of closely interconnected communities, we measure the size of $k$-core ( $k$-core number), where $\mathrm{k}$-core is the maximal subgraph in which each node has at least $k$ connections to other nodes in the subgraph (Kong et al. 2019), or the size of the largest close-knit part of a student's network.

To compute a $k$-core number, the information on the whole school online network is needed. For that, we automatically select all users who indicated on VK that they are studying at the school. This includes both participants and non-participants of the study. We then collect the list of all friends of these users. We anonymized information on any non-participant and analyzed the resulting network. The size of this whole school online network is 670 students. We then computed a $k$-core number for each person's ego network including only their VK friends from the same school.

\section{Offline Social Integration}

We used a number of friends from the same school as a measure of offline social integration. Data on offline friendships were collected using a single name generator. In the survey, participants were asked to indicate up to 10 other students from the same school whom they 
considered friends. Since the sample included only $10 \%$ of all students at the school from different classes, we used unilateral friendship nominations as indicators of friendship, i.e. if at least one person named another as a friend, we considered them friends.

\section{Results}

We find that a higher number of offline friends is associated with severity of depressive symptoms (Pearson's $r=-0.295 ; P=0.002 ; 95 \%$ CI $[-0.461,-0.110]$ ). By contrast, a total number of friends on VK is not correlated with depression severity $(r=-0.096 ; P=0.315$; $95 \%$ CI $[-0.277,0.091])$. One potential reason is that friendship on a social networking site does not necessarily indicate a strong social connection. It is even possible to be "friends" on a social networking site without actually knowing each other.

If we consider separately the number of friends on VK from the same school and not from the same school, we find that the number of online friends from the same school is correlated with depressive symptoms $(r=-0.209 ; P=0.028 ; 95 \%$ CI $[-0.381,-0.023])$ while the number of online friends outside the school is not correlated $(r=-0.084 ; P=0.379 ; 95 \%$ CI $[-0.266,0.103])$. Note that given the small sample size it is impossible to definitively conclude that there is no correlation between the number of online friends outside the school and depression (or between the total number of online friends and depression). However, our results suggest that even if this correlation exists it is significantly weaker than the correlation between depression and the number of friends from the school.

This result might be explained by the important role of the school's local peer community in the structure of adolescent social relations. It is the immediate social environment that a person interacts with on a daily basis. A small number of friends on VK from the same school may be a signal of unpopularity or peer rejection in this major social arena, which can threaten psychological well-being.

Research shows that it is not only the size of adolescents' social network that matters but also belonging to cohesive peer groups (Newman et al. 2007), relationships with which increase markedly in intensity and significance during adolescence (Brown et al. 2004). We analyzed how the size of the largest close-knit group to which the person belongs ( $k$-core number) is associated with depression symptoms.

We find that depressive symptoms are correlated with $k$-core number $(r=-0.204 ; P=0.032$; $95 \%$ CI $[-0,377,-0,018])$ for a person's ego network of their VK friends from the same school. So, the smaller is the dense part of a person's ego network the higher the severity of depressive symptoms. This suggests that it is not the total number of dyadic online relationships that matters but also belonging to a tightly-knit online community.

In addition to online friendship, we consider the intensity of communication in which students engage on the platform, namely exchange of likes. Instead of the total amount of received likes we consider the number of likes per post as a measure of the intensity of communication. This was necessary as people with more pronounced symptoms of depression on average write more posts $(r=0.270 ; P=0.006 ; 95 \% \text { CI }[0.076,0.445])^{2}$. The same has been previously observed in other social networking sites such as Facebook (Frison and Eggermont 2020; Scherr and Brunet 2017).

We find that at the time of data collection there is a significant correlation: participants

\footnotetext{
${ }^{2}$ Because of the skewed distribution, the number of post was log-transformed
} 
with a higher depression score receive on average less likes per post than participants with lower score $(r=-0.31 ; P=0.005 ; 95 \%$ CI $[-0.49 ;-0.10])$. Consistent with the previous results, the association between the number of likes received from online friends outside the school is weaker and does not reach statistical threshold $(r=-0.191 ; P=0.067 ; 95 \% \mathrm{CI}$ $[-0.38 ; 0.01])$. However, six month before the data collection the correlation is non-significant $(r=-0.017, P=0.899,95 \%$ CI $[-0.28 ; 0.25])$.

Thus, consistent with the expectations, the association between likes and depression symptoms is not constant and becomes weaker at time point more distant from the moment of depression measurement.

\section{Conclusions}

The Internet creates unique opportunities for people to connect to each other. Therefore, it might be vastly beneficial for users' psychological well-being as social ties are known to buffer against depression and other mental health issues. However, it is not yet clear whether online interactions are as beneficial for well-being as offline social ties.

In this paper, we have studied associations between online social integration and depressive symptoms in adolescents. We find that the total number of online social ties and the number of online friends outside the school is not associated with depression score. By contrast, online integration into a tightly-knit online community is a protective factor against depression. For school students, this community is online friends from the same school.

SNSs facilitate expansive social networks that grow disproportionately via distant kinds of relationships (Manago et al. 2012). Our results suggest that this expansion of connections may be less beneficial and important in terms of mental well-being than online interactions with people from closer social groups. However, given the small sample size, it is impossible to conclude that these distant relationships are not important at all. Further studies are needed to clarify this question.

The relationship between depression and social integration is temporal in nature because both depression symptoms and social interactions are not constant over time. We have demonstrated that digital traces could facilitate studying this relationship when longitudinal survey data is not available. Specifically, we have employed information on the exchange of likes and have found that the association between the number of likes and depressive symptoms weakens further from the moment of measuring depression. This result could inform future research on the influence of online social integration on psychological well-being.

\section{Acknowledgements}

This work was supported by a grant from the Russian Science Foundation (project №19-1800271).

\section{References}

Appel, H., Gerlach, A. L., and Crusius, J. (2016). The interplay between facebook use, social comparison, envy, and depression. Current opinion in psychology, 9:44-49. 
Berkman, L. and Kawachi, I. (2001). Social ties and mental health. J Urban health, 78(3):458467.

Berkman, L. F. (1995). The role of social relations in health promotion. Psychosomatic medicine, 57(3):245-254.

Blease, C. (2015). Too many 'friends,'too few 'likes'? evolutionary psychology and 'facebook depression'. Review of General Psychology, 19(1):1-13.

Brown, B. B., Larson, J., Lerner, R. M., and Steinberg, L. (2004). Handbook of adolescent psychology. Adolescents' relationships with peers. 2nd. Hoboken, NJ US: John Wiley 86 Sons Inc, pages 363-394.

Burdzovic Andreas, J. and Brunborg, G. S. (2017). Depressive symptomatology among norwegian adolescent boys and girls: the patient health questionnaire-9 (phq-9) psychometric properties and correlates. Frontiers in psychology, 8:887.

Chou, H.-T. G. and Edge, N. (2012). "they are happier and having better lives than i am": The impact of using facebook on perceptions of others' lives. Cyberpsychology, Behavior, and Social Networking, 15(2):117-121.

Costello, D. M., Swendsen, J., Rose, J. S., and Dierker, L. C. (2008). Risk and protective factors associated with trajectories of depressed mood from adolescence to early adulthood. Journal of consulting and clinical psychology, 76(2):173.

Davey, C. G., Allen, N. B., Harrison, B. J., Dwyer, D. B., and Yücel, M. (2010). Being liked activates primary reward and midline self-related brain regions. Human brain mapping, 31(4):660-668.

Durkheim, E. (1897). (1951). Suicide. New York: Free Press.

Elmer, T., Mepham, K., and Stadtfeld, C. (2020). Students under lockdown: Comparisons of students' social networks and mental health before and during the covid-19 crisis in switzerland. Plos one, 15(7):e0236337.

Fatiregun, A. and Kumapayi, T. (2014). Prevalence and correlates of depressive symptoms among in-school adolescents in a rural district in southwest nigeria. Journal of adolescence, $37(2): 197-203$.

Feinstein, B. A., Hershenberg, R., Bhatia, V., Latack, J. A., Meuwly, N., and Davila, J. (2013). Negative social comparison on facebook and depressive symptoms: Rumination as a mechanism. Psychology of Popular Media Culture, 2(3):161.

Ford, E. S., Ahluwalia, I. B., and Galuska, D. A. (2000). Social relationships and cardiovascular disease risk factors: findings from the third national health and nutrition examination survey. Preventive medicine, 30(2):83-92.

Frison, E. and Eggermont, S. (2020). Toward an integrated and differential approach to the relationships between loneliness, different types of facebook use, and adolescents' depressed mood. Communication Research, 47(5):701-728. 
Ganguly, S., Samanta, M., Roy, P., Chatterjee, S., Kaplan, D. W., and Basu, B. (2013). Patient health questionnaire-9 as an effective tool for screening of depression among indian adolescents. Journal of Adolescent Health, 52(5):546-551.

Gonzales, A. L. and Hancock, J. T. (2011). Mirror, mirror on my facebook wall: Effects of exposure to facebook on self-esteem. Cyberpsychology, behavior, and social networking, $14(1-2): 79-83$.

Gunther Moor, B., van Leijenhorst, L., Rombouts, S. A., Crone, E. A., and Van der Molen, M. W. (2010). Do you like me? neural correlates of social evaluation and developmental trajectories. Social neuroscience, 5(5-6):461-482.

Haferkamp, N., Eimler, S. C., Papadakis, A.-M., and Kruck, J. V. (2012). Men are from mars, women are from venus? examining gender differences in self-presentation on social networking sites. Cyberpsychology, Behavior, and Social Networking, 15(2):91-98.

Hobbs, W. R., Burke, M., Christakis, N. A., and Fowler, J. H. (2016). Online social integration is associated with reduced mortality risk. Proceedings of the National Academy of Sciences, 113(46):12980-12984

Holt-Lunstad, J., Smith, T. B., and Layton, J. B. (2010). Social relationships and mortality risk: a meta-analytic review. PLoS medicine, 7(7):e1000316.

House, J. S., Landis, K. R., and Umberson, D. (1988). Social relationships and health. Science, 241(4865):540-545.

Ibrahim, A. K., Kelly, S. J., Adams, C. E., and Glazebrook, C. (2013). A systematic review of studies of depression prevalence in university students. Journal of psychiatric research, $47(3): 391-400$.

Jordan, A. H., Monin, B., Dweck, C. S., Lovett, B. J., John, O. P., and Gross, J. J. (2011). Misery has more company than people think: Underestimating the prevalence of others' negative emotions. Personality and Social Psychology Bulletin, 37(1):120-135.

Kim, J. and Lee, J.-E. R. (2011). The facebook paths to happiness: Effects of the number of facebook friends and self-presentation on subjective well-being. CyberPsychology, behavior, and social networking, 14(6):359-364.

Kong, Y.-X., Shi, G.-Y., Wu, R.-J., and Zhang, Y.-C. (2019). K-core: Theories and applications. Physics Reports, 832:1-32.

Kroenke, K., Spitzer, R. L., and Williams, J. B. (2001). The phq-9: validity of a brief depression severity measure. Journal of general internal medicine, 16(9):606-613.

Kroenke, K., Spitzer, R. L., Williams, J. B., and Löwe, B. (2010). The patient health questionnaire somatic, anxiety, and depressive symptom scales: a systematic review. General hospital psychiatry, 32(4):345-359.

Lee-Won, R. J., Shim, M., Joo, Y. K., and Park, S. G. (2014). Who puts the best "face" forward on facebook?: Positive self-presentation in online social networking and the role of selfconsciousness, actual-to-total friends ratio, and culture. Computers in Human Behavior, $39: 413-423$ 
Leung, D. Y., Mak, Y. W., Leung, S. F., Chiang, V. C., and Loke, A. Y. (2020). Measurement invariances of the phq-9 across gender and age groups in chinese adolescents. Asia-Pacific Psychiatry, 12(3):e12381.

Liu, J., Chen, X., and Lewis, G. (2011). Childhood internalizing behaviour: analysis and implications. Journal of psychiatric and mental health nursing, 18(10):884-894.

Lup, K., Trub, L., and Rosenthal, L. (2015). Instagram\# instasad?: exploring associations among instagram use, depressive symptoms, negative social comparison, and strangers followed. Cyberpsychology, Behavior, and Social Networking, 18(5):247-252.

Manago, A. M., Taylor, T., and Greenfield, P. M. (2012). Me and my 400 friends: The anatomy of college students' facebook networks, their communication patterns, and wellbeing. Developmental psychology, 48(2):369.

Martinez-Pecino, R. and Garcia-Gavilán, M. (2019). Likes and problematic instagram use: the moderating role of self-esteem. Cyberpsychology, Behavior, and Social Networking, $22(6): 412-416$.

Mascheroni, G., Vincent, J., and Jimenez, E. (2015). "girls are addicted to likes so they post semi-naked selfies": Peer mediation, normativity and the construction of identity online. Cyberpsychology: Journal of Psychosocial Research on Cyberspace, 9(1):5.

Nabi, R. L., Prestin, A., and So, J. (2013). Facebook friends with (health) benefits? exploring social network site use and perceptions of social support, stress, and well-being. Cyberpsychology, Behavior, and Social Networking, 16(10):721-727.

Newman, B. M., Lohman, B. J., and Newman, P. R. (2007). Peer group membership and a sense of belonging: their relationship to adolescent behavior problems. Adolescence, $42(166)$.

Okamoto, J., Johnson, C. A., Leventhal, A., Milam, J., Pentz, M. A., Schwartz, D., and Valente, T. W. (2011). Social network status and depression among adolescents: An examination of social network influences and depressive symptoms in a chinese sample. Research in human development, 8(1):67-88.

Qui, L., Lin, H., Leung, A. K. Y., and Tov, W. (2012). Putting their best foot forward: Emotional disclosure on facebook. Cyberpsychology, Behavior, and Social Networking, 15(10):569-572.

Richardson, L. P., McCauley, E., Grossman, D. C., McCarty, C. A., Richards, J., Russo, J. E., Rockhill, C., and Katon, W. (2010). Evaluation of the patient health questionnaire-9 item for detecting major depression among adolescents. Pediatrics, 126(6):1117-1123.

Rosenquist, J. N., Fowler, J. H., and Christakis, N. A. (2011). Social network determinants of depression. Molecular psychiatry, 16(3):273-281.

Rubin, K. H., Bukowski, W. M., and Parker, J. G. (2007). Peer interactions, relationships, and groups. Handbook of child psychology, 3 .

Scherr, S. and Brunet, A. (2017). Differential influences of depression and personality traits on the use of facebook. Social Media + Society, 3(1):2056305117698495. 
Seabrook, E. M., Kern, M. L., and Rickard, N. S. (2016). Social networking sites, depression, and anxiety: a systematic review. JMIR mental health, 3(4):e5842.

Seeman, T. E. (1996). Social ties and health: The benefits of social integration. Annals of epidemiology, 6(5):442-451.

Steers, M.-L. N., Wickham, R. E., and Acitelli, L. K. (2014). Seeing everyone else's highlight reels: How facebook usage is linked to depressive symptoms. Journal of Social and Clinical Psychology, 33(8):701-731.

Thoits, P. A. (2011). Mechanisms linking social ties and support to physical and mental health. Journal of health and social behavior, 52(2):145-161.

Tsai, F.-J., Huang, Y.-H., Liu, H.-C., Huang, K.-Y., Huang, Y.-H., and Liu, S.-I. (2014). Patient health questionnaire for school-based depression screening among chinese adolescents. Pediatrics, 133(2):e402-e409.

Tsehay, M., Necho, M., and Mekonnen, W. (2020). The role of adverse childhood experience on depression symptom, prevalence, and severity among school going adolescents. Depression research and treatment, 2020.

Ueno, K. (2005). The effects of friendship networks on adolescent depressive symptoms. Social Science Research, 34(3):484-510.

Umberson, D., Crosnoe, R., and Reczek, C. (2010). Social relationships and health behavior across the life course. Annual review of sociology, 36:139-157.

Vogel, E. A., Rose, J. P., Roberts, L. R., and Eckles, K. (2014). Social comparison, social media, and self-esteem. Psychology of Popular Media Culture, 3(4):206.

Young, C. B., Fang, D. Z., and Zisook, S. (2010). Depression in asian-american and caucasian undergraduate students. Journal of affective disorders, 125(1-3):379-382.

Zell, A. L. and Moeller, L. (2018). Are you happy for me... on facebook? the potential importance of "likes" and comments. Computers in Human Behavior, 78:26-33.

\author{
Affiliation: \\ Elizaveta Sivak \\ HSE University \\ Moscow, Russia \\ E-mail: esivak@hse.ru \\ Julia Dementeva \\ HSE University \\ Moscow, Russia \\ E-mail: yudementeva@hse.ru
}


Ivan Smirnov

HSE University

Moscow, Russia

E-mail: ibsmirnov@hse.ru 